\title{
TELECOMMUNICATIONS AND RADIO ENGINEERING
}

Volume 60, Numbers 1-12, 2003

Volume 60, Number 1\&2, 2003

\section{WAVE PROPAGATION AND SCATTERING}

Yu.F. Logvinov Sea Surface Statistics in the Case of Radio Wave Backscattering at Low Grazing Angles

V. A. Komyak and S. A. Shilo Effect of a Rough Sea Anisotropy on Angular Dependences of the Ocean Microwave Radiation 1

A.A. Kirilenko, D.Y. Kulik, and V.I. Tkachenko General Scheme of the Mode-Matching Technique as a Basis for Generalized Solutions to the Internal Boundary-Value Problems ... ......23

S.F. Kulishenko, A.A. Kirilenko, and S.L. Senkevich Waveguide Band Matched by the Stepped 34 Miter

V.A. Doroshenko and E.K. Semenova Field Behaviour Near the Vertex of a Cone with

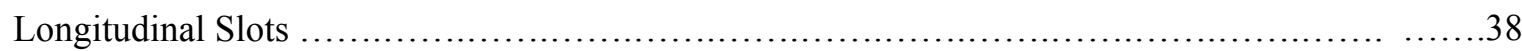

V.S. Miroshnichenko and Ye.B. Senkevich Experimental Determination of Equivalent Circuit Parameters of an Open Resonator Coupled to Transmission Lines ...................................45

Z.E. Eremenko and E.M. Ganapolskii Hemispherical Cavity Resonator for Measuring of Permittivity of Strongly Absorbing Liquid in Small Volume

\section{MICROWAVE AND QUANTUM ELECTRONICS}

M.A. Kopot, A.N. Nikitenko, and G.I. Churyumov Investigations into the Dispersion Characteristics of the Electrodynamic Systems with Resonators of Complex Shape

V.B. Baiburin, A.V. Sysyev, V.A. Sysyev, and A.A.Terentiev Interaction of Axial and Cyclotron Electronic Oscillations in M-Type Devices

A.V. Vasyanovich, A.V. Gritsunov, A.N. Nikitenko, and M.O. Horunzhii General Principles of Spectral Modeling of Microwave Devices

G.P. Ermak, V.P. Kochergin, and P.V. Kupriyanov Millimeter-Wave Planar Frequency Multiplier 3 
U.R. Liyepin' and O.N.Stavitsky Algorithmic Reconstruction of the Radiation-Pattern Characteristics of a Partially Unexcited Antenna Array

V.V. Ovsyanikov and Ye.D. Romanenko Radiation Characteristics of Circularly Polarized Dipole Antenna Arrays ...

V.A. Katrich and M.V. Nesterenko The Near-Zone Field and Resonant Frequencies of Narrow Longitudinal Slots in the Broad Face of a Rectangular Waveguide

\section{RADAR REMOTE SENSING}

O.V. Sytnik, Ye.I. Myroshnychenko, and Yu.A. Kopylov Estimation of Implementation Errors Effect on Characteristics of Pseudorandom Radar Signal

A.V. Sokolov, and V.V. Lukin Local Recognition of Radar Images by Three-Layer Neural

Network Using Spatial Features ....

SIGNAL PROCESSING

V.A. Pismenetski, N.I. Sliptchenko, and P.I. Platonov The Dispersion-Spectral Method for Identification of Pulsed Radio Signals

L.P. Yatsuk Potential Functions in the Problem of Excitation of a Waveguide Filled with a ThreeLayered Dielectric by a Longitudinal Magnetic Current

L.B. Minakova and L.A. Rud Algorithms of Analysis and Synthesis of Rejection Cells Based on the Partially Filled H-Plane Stubs in Rectangular Waveguide

Ye.M. Ganapolskii Broadband Echo-Box of Millimeter Wave Band for a Long-Time Delay of a Microwave Pulse Signal

V.S. Litvinenko, Yu.V. Prokopenko, and Yu.F. Filipov Surface Electromagnetic Oscillations in a Semiconductor Sphere

\section{ANTENNAS AND FEEDER SYSTEMS}

N.N. Gorobetz and N.P. Yeliseyeva Radiation Characteristics of an Electric Dipole of Arbitrary Orientation Placed Above a Plane Screen

Yu.V. Kornienko Construction of Non-Redundant Antenna Configurations on a Hexagonal Mesh by a Random Search ....

Y.Y. Volkolupov, M.A. Krasnogolovets, O.G. Lebedev, M.A. Ostrizhnoy, and V.I. Chumakov On the Determination of Time Characteristics of Radiating Antennas .........

P.A. Melyanovsky and I.V. Popov Helical Antenna with Transversal Radiation .66

V.V. Popovskiy and Yu.Yu. Kolyadenko Adaptive Interference Compensators: Problems and 
Methods of Their Solution

I.D. Gladkoskok and V.A. Nazarenko Field of Fresnel Zone-Focused Linear Antenna Array with

Random Errors of Excitation and Element Position .78

PHYSICAL PROCESSES IN ELECTRON DEVICES

V.D. Naumenko, A.N. Suvorov, and A.R. Syrov Investigating the Temperature Regime of the Starter Cathode in a Millimeter Wave Magnetron ................................................89

K.A. Lukin and P.P. Maksymov Electrostatic Fields in Reverse-Biased $p n-i-p n$ Structures ..............93

D.V. Snezhko and N.N. Roshytsky Optochemotronic Sensors: New Elements for Biomedical Diagnostic Systems. 1. Basic Concepts, Theoretical Description, Physical and Mathematical Models of the Processes in Optochemotronic Sensors

\section{QUANTUM ELECTRONICS}

V.V. Shevchenko Electrical and Spectral Matching in Lamp Pumping System of Pulsed Lasers ... ......112

V.V. Shevchenko Holographic Interferometry of a Lateral Surface of KDP Crystals and

Modulation of a Laser Radiation at Resonance Frequencies

\section{APPLIED RADIO PHYSICS AND INDUSTRIAL ELECTRONICS}

P.K. Nesterov Quasi-Optical Interferometers with Enhanced Phase Sensitivity

V.I.Borsch, V.V.Koval, and B.Ya Kostik. Oscillations in Optimal-High-Speed Radioautomatics Systems

N.D. Koshevoy, V.A. Gordienko, O.N. Koshevoy, and T.G. Rozhnova Comparative Analysis of Pressure Sensors with Digital Output

Volume 60, Number 5\&6, 2003

\section{MICROWAVE ELECTRODYNAMICS}

D.Yu. Kulik, V.I. Tkachenko, and S.L. Senkevich TEM-Field Calculation for Waveguides with Complicated Cross-Sections ....

T.L. Zinenko E- and H-Polarized Plane Waves: Scattering and Absorption by an Impedance Strip Grating

A.V. Gribovsky Properties of Reflection Grating Composed of Short-Circuit Rectangular

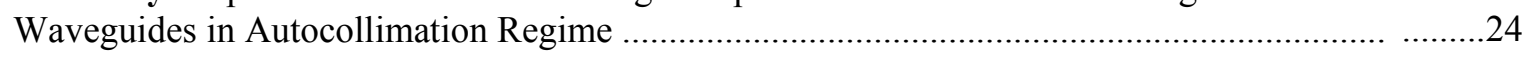

Yu.V. Svishchev The Vertical Electric Dipole Field in Presence of a Sphere with Circular Hole .... ..........31

L.P. Yatsuk and T.G. Nazarenko Scattering from the Slots in a Waveguide Filled with an Imperfect Dielectric

V.D. Sakhatsky and A.R. Korsunov New Principles of Development of Corner Filters .52 
T.N. Ogurtsova, G.P. Pochanin and P.V. Kholod A Receiving Loop Antenna for Super-

Broadband Pulsed Signals

N.N. Gorobets, V.I. Chebotaryov, and G.M. Chekalin Synthesis of the Specified State of

Electromagnetic Wave Polarization in the Arbitrary Elliptically Polarized Basis .............................71

V.V. Ovsyanikov On the Principles of Calculating Radiating Devices with Impedance Elements ... .........82

\section{TELECOMMUNICATION AND SIGNAL PROCESSING}

N.M. Kalyuzhny, S.V. Pshenichnykh, I.V. Puziy, and A.A. Asanov Maintenance of the Continuity of a Phase When Forming Pulse Radio Signals with LFM Transformation

V.N. Zaharchenko, V.V Korchinsky, and A.A. Grin' The Efficiency of Data Transmission Systems with the Decision Feedback and Variable Parameters of the Correcting Code

Y.P. Kunchenko and T.V. Prokopenko Application of the Polynomial Maximization Method for Estimation of Signal Parameters Received by the Antenna Arrays

\section{SOLID-STATE RADIO PHYSICS AND PLASMAS}

Ye.L. Cherkashina Selectivity Characteristics of the First and Second-Order Bragg Diffraction Components During Interaction of Light with a Transverse Ultrasonic Wave in Single-Axis Crystals .110

S.Yu. Karelin Parameters Measurements of Semimagnetic Semiconductors by Means of the Electron Paramagnetic Resonance Method

A.A. Barannik, S.A. Bunyaev, and N.T. Cherpak Accuracy and Sensitivity of Surface Resistance Measurements of High-Temperature Superconductor Films Using Quasioptical Dielectric Resonators

D.L. Grekov, N.A. Azarenkov, A.A. Bizyukov and V.P. Olefir An Optimized Antenna for Plasma Generation in the ПР-1 Facility

\section{PHYSICAL PROCESSES IN ELECTRON DEVICES}

O.V. Botsula and E.D. Prokhorov Joint Operation of Two-Level Resonant Tunnelling and Gunn Diodes

V.I. Afanas'iev Magnetostatic System for Electron Beam Control

M.I. Ayzatsky, I.V. Khodak, V.A. Kushnir, V.V. Mitrochenko, and V.F. Zhiglo Ultra-High

Frequency Intense Pulse Electron Sources

Volume 60, Number 7, 8, and 92003 
A.E. Svezhentsev Modeling a Periodic Array of Radiators on a Cylindrical Surface ..... 1

A.I. Kozar Electromagnetic Wave Scattering in a Waveguide Containing Homogeneous Magnetodielectric Spheres ......

Yu.V. Prokopenko and Yu.F. Filippov Influence of the Finite Conductivity of the End Walls on the Spectrum and Power Characteristics of the Anisotropic Dielectric Circular-Disk Resonator

N.I. Ayzatsky and Ye.Yu. Kramarenko Coupled Oscillator Model in Theory of Layered Dielectric

I.Yu. Vorgul and A.V. Vorgul Solving Electrodynamics Problems in Time-Varying Media:

Different Approaches Based on the Integral Equation

A.A. Barannik, Yu.V. Prokopenko, T.A. Smirnova, Yu.F. Filipov, and N.T. Cherpak

A Quasi-Optical Dielectric Ring-Resonator With Conducting Endplates

V.K. Kiseliov and S.V. Mizrakhi Quasi-Optical Sectional Waveguide Taper for Submillimeter Wave Band

V.I. Gomozov, A.V. Gomozov and S.V.Titov Space-, Phase- and Frequency-Based Control of Signal Focusing in Planar Phased Arrays with a Discrete V-Shaped Distribution of Carrier Frequencies

V.A. Doroshenko and Y.K. Semenova Electromagnetic Wave Diffraction by a Cone with Longitudinal Slots and a Solid Conic Screen Inside

V.V. Ovsyanikov, A.V. Reuta, and Ye.P. Beznosova Analysis and Optimization of a TwoFrequency Turnstile Radiator with Impedance Elements in the Branches . .76

\section{TELECOMMUNICATIONS AND SIGNAL PROCESSING}

V.Yu. Murashev, A.V. Kublik, I.A. Sukhoivanov Calculation of Four-Wave Mixing Power Arising in Fiber-Optic Communication Lines with Wavelength-Division Multiplexing

A.I. Filipenko Determining of Refractive Index Profile of Optical Fibers from Measured Intensity in Near-Field Zone ....

G.V. Pevtsov and D.A. Kolisnichenko Quality Indexes for Optimization of the Multifunction Wireless Devices

V.A. Omel'chenko, V.V. Balabanov and B.M. Bezruk Multicriterion Problem on Detection of Unknown Random Signals Using the Spectral Method

D.V. Bondar, A.N. Zelenin, and A.I. Kostromitsky The Analysis of the Active Corrector of Frequency Characteristics 
V.A. Abdulkadyrov, E.M. Khutoryan, and A.I. Tsvyk Interaction of a Plane Electromagnetic Wave with a Semiconductor-Dielectric Structure Containing a Diffraction Grating ....

Yu.O. Averkov and V.M. Yakovenko Excitation of Surface Electromagnetic Wave Pulses by

Electron Bunches Intersecting a Metal-Dielectric-Semiconductor Structure

\section{PHYSICAL PROCESS IN ELECTRON DEVICES}

O.V. Zemlyaniy and K.A. Lukin Correlation-Spectral Properties of Chaos in the Nonlinear

Dynamical System with Delayed Feedback and Asymmetric Nonlinear Map

A.A. Manko Measurement of the Frequency Switching Time of Microwave Oscillators

\section{MEASUREMENT SYSTEMS}

A.F. Velichko, D.A. Velichko, and V.M. Shokalo Spatial-Temporal Filtering in Precision Radio-

Engineering Systems of Technical Diagnostics

O.A. Remayeva, Ye.V. Remayev, and G.K. Snurnikova Error Estimate in Refraction

Corrections for the Space Object Coordinates determined by Electromagnetic Waves

Yu.Ye. Gordienko and A.Yu. Panchenko Modulation Conversions of Information Signals in

Resonance Gauges of Parameters of Objects and Mediums

V.I. Lutsenko, Ye.N. Belov, I.V. Lutsenko, and S.I. Khomenko The Troposphere Refraction Estimation by Attenuation Factor of Radiowave beyond-the-Horizon Propagation

I.M. Mytsenko and S.I. Khomenko Diagnostics of Microwave Propagation Conditions Through the use of Signal Reflection from Sea Surface

STATISTICAL RADIO PHYSICS

O.V. Sytnik Invariant Transformation in Identification Theory .20

\section{TELECOMMUNICATIONS AND SIGNAL PROCESSING}

B.M. Bulgakov, S.A. Kravchuk, T.N. Narytnik, V.V. Popovsky, and S.I. Tatarchuk The Concept of Application of Millimeter and Submillimeter Electromagnetic Waves in Telecommunication Information Systems .34

I.P. Panfilov and A.A. Scopa Reliability of the Communication Link Consisting of Primary and Reserve Channels

A.O. Puzanov Some Properties of Videopulse Soil-Layer Scattering with Continuous Variation in 
Frequency-Dependent Electrical Characteristics

V.B. Razskazovsky and Yu.A. Pedenko Comparison of Elevation Angle Measurement

Techniques over the Sea

V.N. Gorobets, V.G. Gutnik, and F.V. Kivva Experimental Doppler Spectra of Radiolocation

Signal Reflections from Fallen Ship Waves

\section{APPLIED RADIO PHYSICS: SPACE, ATMOSPHERE AND EARTH SURFACE RESEARCH}

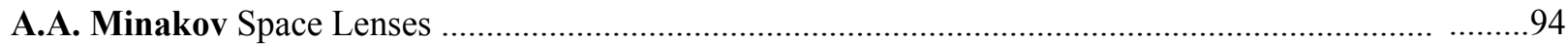

V.G. Galushko and G.V. Lytvynenko Application of the Frequency-and-Angular Sounding

Technique to Recovering the Three-Dimensional Electron Density Distribution in the Ionosphere

A.P. Nickolaenko, L.M. Rabinowicz, A.V. Shvets and, A.Yu. Schekotov Detection of Splitting

of Shumann Resonance Eigenfrequencies

A.M. Gokov Development of the Method of Determining the Electron Molecule Collision Frequencies in the Ionospheric D-Region by the Partial Reflection Technique .....

A.M. Gokov Simultaneous Determination of Electron Density and Electron-Neutral Molecule Collision Frequencys in the Ionospheric D-Region by a Partial Reflection Technique

A.M. Gokov and O.F. Tyrnov Peculiarities of the Middle Latitude Ionospheric D-Region Dynamics, Caused by the Solar Terminator

I.G. Zakharov and M.V. Lyashenko Comparison of the Experimental and Model Values of Total Electron Content in the Ionosphere above Eastern Europe . 